Organizing the Tasks in Complex Design Projects

Steven D. Eppinger

Daniel E. Whitney

Robert P. Smith

David A. Gebala

WP\# 3083-89-MS

October 1989

(revised June 1990) 


\title{
Organizing the Tasks in Complex Design Projects
}

\author{
Steven D. Eppinger \\ Daniel E. Whitney \\ Robert P. Smith \\ David A. Gebala \\ Massachusetts Institute of Technology
}

\begin{abstract}
This research is aimed at organizing complex design projects in order to perform faster product development. We use a variation of Steward's design structure matrix [32] to represent both the sequence of and the technical relationships among the many design tasks to be performed. These relationships define the "technical structure" of a design project. We will develop analytical techniques to find alternative sequences and definitions of the design tasks, offering opportunities to speed development progress by improved coordination and information transfer. Our algorithms will consider the technical structure of a project in order to identify which tasks should be resequenced and which tasks should be redefined or split into smaller sub-tasks to avoid development bottlenecks. We will develop these techniques into a strategy for design management which can significantly reduce the overall project complexity by reorganizing the few critical tasks. We expect that this research will benefit not only new design tasks that have never been structured before but also longstanding, often repeated design tasks that may have drifted into poor organizational patterns over many years.
\end{abstract}

\section{Introduction}

Intense competition forces firms to develop new products at an increasingly rapid pace. This mandate places substantial pressure on engineering teams to develop products better and at the same time develop products faster. The organizational responses to these two challenges are, respectively, "design for manufacture" and "simultaneous engineering".

\section{Acknowledgment:}

This research is funded by the MIT Leaders for Manufacturing Program, a partnership involving eleven major US manufacturing firms and MIT's engineering and management schools.

The authors are affiliated with (respectively) MIT Sloan School of Management, Charles Stark Draper Laboratory, MIT Sloan School of Management, and MIT Department of Mechanical Engineering. 
Design for manufacture (DFM) is the adoption of a new attitude among engineers that emphasizes the important manufacturing issues early in the product development process [11]. Simultaneous engineering is an effort to create the product design and the manufacturing process concurrently by allowing design engineers to work closely with manufacturing engineers, field service engineers, and representatives of others interested in the manufacture and use of the product [19]. While these techniques are often difficult to practice, firms using them claim to be designing better products in less time as a result of increased coordination and awareness of broader design issues [39].

Despite these successes, complex product development efforts remain a technical and organizational challenge. The design of an automobile, aircraft, or computer can involve coordinating hundreds or thousands of engineers making more than a million design decisions over months or years. None of these many tasks is performed in isolation $[9,10]$. Each design choice may be a tradeoff affecting many other design parameters. Facilitating the transfer of information among design groups is an essential organizational task of product design managers $[2,12,22,37]$.

For the engineers, however, the challenges of simultaneous engineering are particularly difficult when many design tasks are interdependent and cannot be performed in series or in parallel. In these cases, since many design decisions are coupled, they must be made "simultaneously", perhaps by iteration or negotiation among specialists [7]. Potentially many engineers from various disciplines must be involved in this complex decision process $[15,30]$. Strategically decoupling the major design tasks into sub-systems can reduce the sizes of the working design groups, and this can have a dramatic impact on development performance [3, 22].

This research builds on work by Steward [32,33] that is introduced below. We are investigating strategies for design management that can reduce the overall product development time and improve the quality of design decisions. In many industries, the development process can be clearly mapped out in terms of the major design milestones and the minor negotiations which must take place along the way $[5,17]$. Some examples can be found in aspects of automotive, computer, and aircraft design. We contend (as Simon also argues [28]) that design procedures can be documented and studied; then they can be greatly improved. By analyzing the complex relationships among design decisions, we can find the "design drivers" and the "decoupling points". These concepts are central to the development of strategies to better organize the design tasks and improve coordination among the designers, facilitating faster and better product development. 
We aim to develop two major results, a computer-based analysis tool and a set of organizational strategies. Together these can improve the product development process by:

1. identifying the key factors which determine many of the design results,

2. reducing both the perceived and actual complexity of design tasks,

3. sharing engineering data earlier and/or with known confidence,

4. redefining critical tasks to facilitate overall project flow,

5. forcing designers to organize their decision processes, and

6. helping design managers to place emphasis on facilitating coordination.

Two types of design can be distinguished: design of entirely new items, for which there exists at first no organized design procedure; and redesign of existing items, such as automobiles, where there is a large investment in existing design procedures, often heavily bureaucratized. The need for aids such as proposed here is easily recognized in the first case. The benefit of this work for the second type may be just as great but harder to recognize. This is due to the fact that a "procedure" exists and seems to work well. However, it may have grown up organically and historically and may never have been subjected to careful analysis. So its internal inefficiencies or irrationalities remain undetected.

Our approach involves mapping an existing or proposed design procedure into an array representing the inter-relationships among the many design tasks which must be accomplished. The argument for a design methodology which corresponds to the underlying structure of the design problem has been articulated by Steward [32], Simon [28], and other authors, most notably Alexander in the 1960s [1]. The analysis we will perform considers the relative importance of each design parameter to other parameters, allowing the information requirements to determine the appropriate scheduling of the decisions. The result of this analysis is an array of options for a manager or engineer to rearrange or reprioritize the tasks. Strategies include decoupling and resequencing tasks, insertion of new decision points, splitting or condensing tasks, and other schemes to improve the flow of information and decisions.

The next section discusses tools to aid in design project management and explains the formulation of a task matrix representation. We then show how we use analytical techniques to find improved design sequences and project management strategies.

\section{Design Organization Techniques}

The design management response to the challenge of reducing product development lead time has typically been to encourage engineers to develop the product and its associated manufacturing process concurrently. This policy has two beneficial effects. First, it emphasizes the need for design engineers to be 
aware of production issues, and this is the focus of the popular "design for manufacturing" approach. Second, we find that designers are sharing or transferring information to their counterparts in manufacturing engineering much sooner than they had done previously.

These trends bring up many new issues in design project management. Today we find that the design procedures which have evolved over the years are being transformed into new design recipes. Engineers are now performing their standard design functions with potentially different inputs and outputs. They are expected to make use of more information than was previously available and to practice new design methodologies $[6,11,14,34]$. This new information takes many forms, including more accurate consumer preference data and manufacturability data. Distribution of the results of their engineering efforts is also more broad in scope. Designers are now expected to work more closely with manufacturing and marketing, reporting on their progress at frequent intervals.

Engineers and managers have adopted techniques for planning, organizing, and monitoring the complex network of tasks in a large development project. These procedures require documentation of the entire design process. However, the most widely used representations do not adequately describe the design structure as we define it.

The most popular project planning tools [42] use network diagrams to represent the precedence relationships among activities. In the PERT method, three time estimates are given to each task (optimistic, pessimistic, and a best guess). Probability of timely task completion can be computed along with the associated start times for each activity. The critical path method (CPM) performs a linear time/cost tradeoff for tasks on the critical (longest-lead-time) path. To accelerate the project, additional resources (at greater costs) are placed on critical tasks to shorten the critical path. (The program evaluation review technique (PERT) was developed in the 1950s for the Polaris project, which involved scheduling over 3000 tasks. PERT is credited with supporting completion of the project up to two years ahead of schedule. The critical path method (CPM) was developed at Du Pont.)

The precedence diagramming method (PDM) [41] places the activities on the nodes of the graph, rather than the arrows, and this allows the graph to be drawn to scale, visually representing time. All of these network techniques require that there be only one-way progression along paths, with no feedback or iteration, no feed-forward of information part-way through a task, etc. The emphasis is placed on the interactions between the tasks, not on the details within the tasks. The tendency is therefore to define tasks in the large, ignoring a multitude of engineering interactions required within each one.

A more powerful technique is the Air Force standard IDEFo project definition method [18, 26, 27], which can be used to represent some of the intra- 
task complexity. (IDEFo was developed by Ross at Softech, Inc. in the 1970s. The technique was known as SADT until it was adopted by the Air Force as a standard representation.) Moreover IDEF0 supports the concepts of "as is" and "should be" design procedures but provides no method for moving from the former to the latter. $\mathrm{IDEF}_{0}$ charts tend to grow rapidly for large tasks until managers can hardly see what is going on. This complexity is managed by using available software for describing and analyzing the diagrams [13]. Many firms (including US automotive and aircraft manufacturers) are using such tools.

Project management tools are generally applied to the design process on the basis of "start task/complete task" representation, ignoring the technical structure of the tasks, the information tasks need or produce, and the overall information flow network that underlies the whole effort. Also, they may document an idealized view of the process that ignores the vital, informal, and usually undocumented interactions that are undeniably essential to project success.

When a representation omits significant effects, then important system behavior remains unexplained. If we ignore the design task coupling, then we will fail to recognize the most significant challenges of the design effort. On the other hand, if an improved representation technique can include the complex task dependencies inherent within the process, then those relationships can finally be studied and exploited if possible. We propose that new approaches to managing design complexity will become practical through the use of a more accurate description of the design process.

\section{Sequencing Design Tasks}

Creating the more detailed description we seek involves explicitly mapping out the technical aspects of the design procedure. We contend that to be most useful, the design representation must include not only the sequence of the tasks but also the many technical relationships among the tasks. The description we use is based on Steward's design structure matrix. However, before presenting this method, we will illustrate some general issues in sequencing design tasks and transferring engineering information.

Consider two design tasks, labelled $\mathbf{A}$ and B. Figure 1 shows directed graphs (digraphs) [38] of three possible ways in which the two can be related. If task B simply requires the output of task A (or vice-versa), then the two tasks are dependent and are typically done in series. On the other hand, the two would be entirely independent if tasks A and B could be performed simultaneously with no interaction between the designers. Finally, if task $\mathbf{A}$ needs information from task $B$, and also task B requires knowledge of task A's results, then the two tasks are interdependent. 


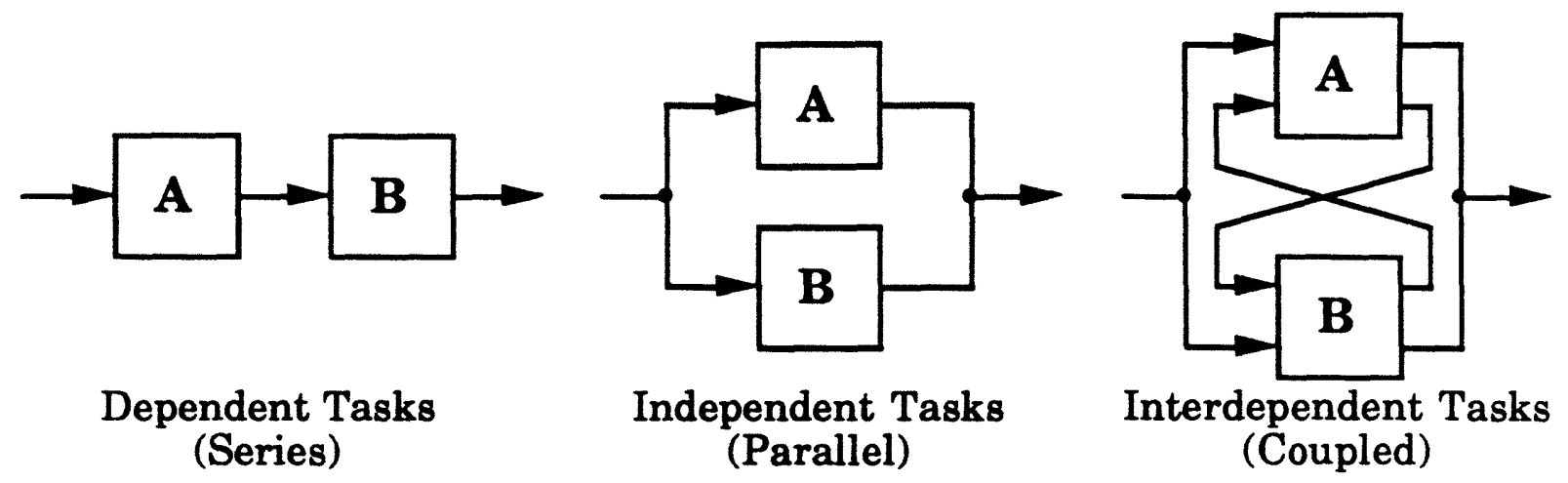

Figure 1. Three Possible Sequences for Two Design Tasks

Coordinating either the dependent (series) tasks or the independent (parallel) tasks is quite straightforward. Certainly with no limitation on resources, the parallel tasks can be completed more quickly. The interdependent (coupled) tasks are much more challenging to organize, often requiring much more design time and many iterations of information transfer [35].

To illustrate using a familiar theme, we can envision task $\mathbf{A}$ to represent a product design function, and task $B$ to represent a product manufacturing function. Then our series model depicts the outdated "throw the design over the wall" methodology. The parallel tasks model might then represent an idyllic view of simultaneous engineering, where both design and manufacturing functions are given the same challenge, and they magically develop product and process concurrently (without complex interactions). The coupled tasks model is a more realistic diagram of simultaneous engineering, where the information transfer is essential and iteration is typical.

PERT software tools can typically analyze project sequence diagrams only if they contain no coupling (loops). The representation requires the coupled tasks to be bundled into larger design tasks. If the project planner chooses to consider the tasks separately, then the essential information coupling must be neglected. We now present a representation which handles the complex relationships among tasks more naturally.

\section{The Design Structure System}

Steward's design structure system [32, 33] uses a representation which allows the direct coupling of any task to another. Figure 2 shows the design structure matrix in its original binary form as described by Steward, where the design tasks to be performed are each represented by an identically labeled row and column of the matrix. The marked elements within each row identify which other tasks must contribute information for proper completion of the design. For example, the mark in $<$ row $\mathbf{D}$, column $\mathbf{L}>$ indicates that completion of task $\mathbf{D}$ 
requires information to be transferred from task $L$. We would then desire task $L$ to be performed before task $\mathbf{D}$.

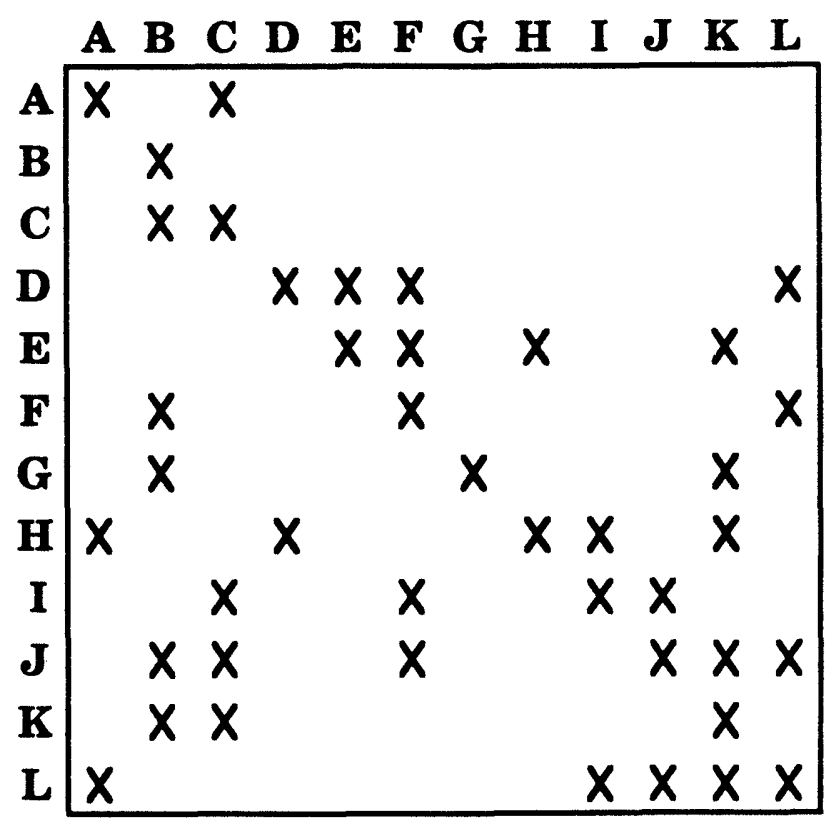

Figure 2. A Binary Design Structure Matrix, Unpartitioned

The primary goal of design structure management is to find a sequence of these design tasks which allows this matrix to become lower triangular. If the tasks can be sequenced so that each one begins only after it receives all the information it requires from its predecessors, then there is no coupling remaining in the design problem. However, this rarely happens. Instead, we find something like Figure 3, which shows the matrix representing the same problem after the tasks have been rearranged (partitioned) by interchanging tasks (swapping rows and the corresponding columns) to achieve a more organized design sequence.

The partitioning process has sequenced the tasks to be performed in the order: B-C-A-K-L-J-F-I-E-D-H-G. The matrix shows that task $\mathbf{C}$ is dependent upon task B, so they are performed in the sequence B-C. Tasks $A$ and $\mathbf{K}$ can be then be completed in parallel (since task $\mathbf{K}$ does not depend upon task $\mathbf{A}$ ). The two "blocks" encompassing the task sequences L-J-F-I and E-D-H identify two sets of coupled tasks, the most challenging aspects of this design problem. Here are tasks to be performed simultaneously or group-wise, and the information transfer required may take the form of iteration and/or negotiation. Steward's work in this area ends here with a procedure for working through the loops in the coupled tasks. Other schemes for identifying the blocks have also been found [16]. 


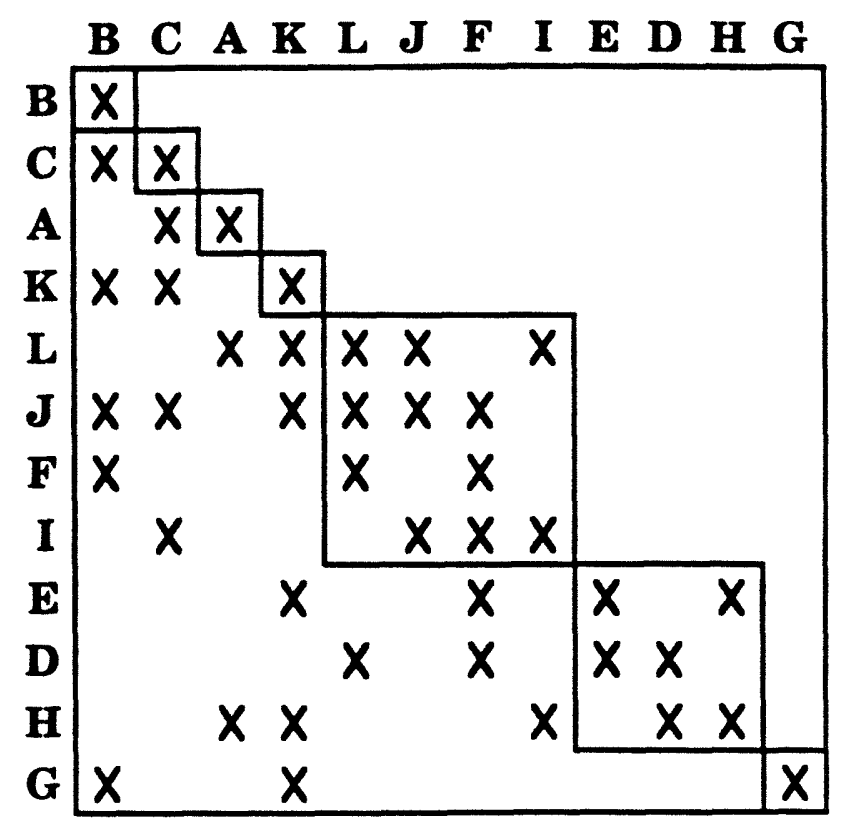

Figure 3. The Binary Design Structure Matrix, Partitioned

When the design structure matrix cannot be manipulated into lower triangular form, we then seek a form that minimizes the size and number of the remaining blocks on the diagonal. Collapsing these blocks into single tasks (as would be required for PERT analysis) would certainly make the project appear to be simpler. In our example, we would combine tasks $L, J, F$, and I into one task and then collapse tasks $\mathbf{E}, \mathbf{D}$, and $\mathbf{H}$ into another. We would be left with seven tasks in lower-triangular form instead of the twelve tasks as shown. However, this approach hides the real design problems and precludes any opportunity to further improve the design procedure by applying other techniques.

The partitioned matrix in Figure 3 is not unique, but rather its form depends on the algorithm used to reorder the tasks. Recent work at NASA [24, 25, 30] has implemented Steward's representation using a rule-based (expert system) partitioning algorithm. As an example problem, they model the process of designing a complex spacecraft antenna system with over 50 interrelated tasks [20]. The design structure analysis showed that in this design problem there is a small number of large subsystems containing from 5 to 20 tasks each. These coupled groups of tasks are then performed in the sequence: actuators, sensors, structures, dynamics, controls, etc.

As presented by Steward and in the NASA work, the binary design structure matrix only represents strict precedence relations. (A task either does or does not depend upon another task.) In complex design problems, we may find that the binary matrix is crowded with weak dependencies. This would lead to an extremely coupled design matrix. 


\section{Our Approach to Designing Design}

Design procedures need to be designed. Problems are complex, people may lose sight of the goal of the whole process, and opportunities for making the process efficient may be hard to recognize. This work establishes a design procedure design method with five steps, listed below, and the remainder of this paper details our progress in implementing this scheme.

1. Make the mathematical or engineering model of the system to be designed.

2. Make a decision structure model, showing options for linking decisions and subproblem elements to each other.

3. Make a decision sequence model, indicating which design decisions should be first, second, etc., to make the process as informative and efficient as possible.

4. Make a sensitivity model, in which the robustness of the decision sequence can be evaluated in terms of the effect of incorrect specifications or changes in specs on the progress of the design process.

5. Make a simulation model of the decision sequence process in order to time it, observe it iterate, and seek improvements through further resequencing.

\section{Extensions to the Design Structure Matrix Representation}

We extend the basic representation by explicitly including measures of the degree of dependence, so that we can develop more sophisticated analytical procedures to further improve the design process. Figure 4 shows a numerical design structure matrix which uses values to represent the importance of each task dependency. The rules for partitioning this matrix can now consider rearranging tasks to (for example) minimize the importance of the above-diagonal elements. This would allow even the iteration within the coupled sub-systems to be minimized since the most important inter-task relationships are in the proper positions.

We are developing analytical procedures which take advantage of numerical design structure matrix representations such as this one. The numerical values need not necessarily depict the strength of the organizational dependency. Other metrics to consider include task completion time, functional coupling [23], historical variance of task results, certainty of planning estimates, or volume of information transfer. 


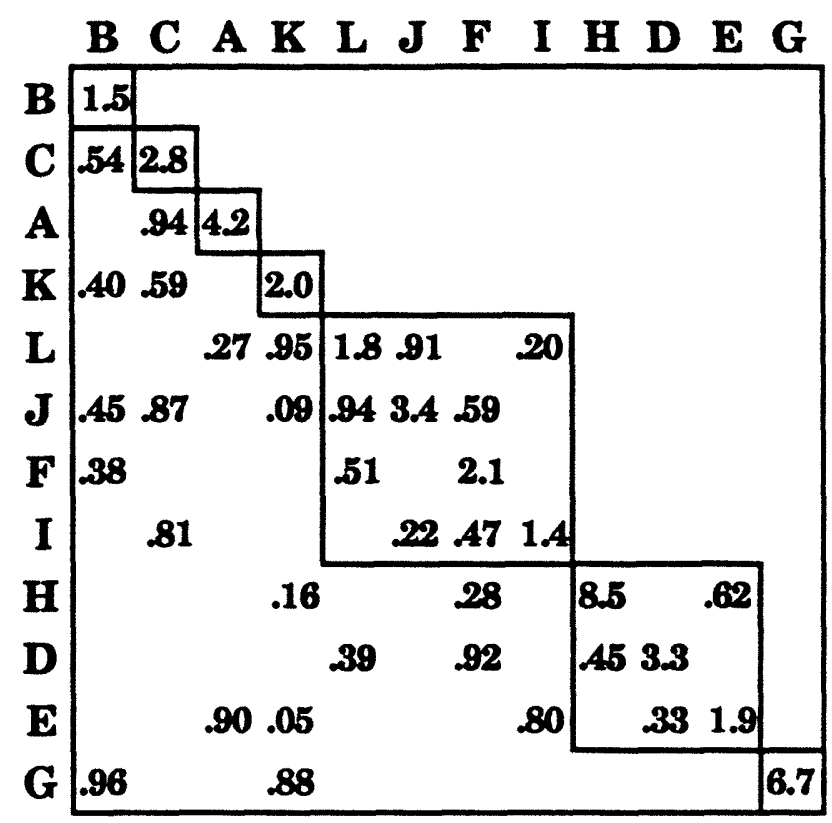

Figure 4. A Numerical Design Structure Matrix

For example, if a task vitally depends on information from another task but that information is known to lie within predictable limits, then the dependent task might be able to start based on a good guess of the anticipated information. Thus the dependency would be represented as weak. Similarly, if the task depends only slightly on information that is largely unpredictable, the dependency might again be judged as weak. Contrarily, needed information with large impact and large variability implies a strong dependency. (We cannot start without it, nor can we predict it well enough.) An "importance ratio" can be calculated as the basis for determining the strength of the dependency. This ratio would be similar in definition to Taguchi's "signal-to-noise" ratio used to compare the relative effects of parameters [8].

Furthermore, each matrix element could instead be a vector of many such measures, such as certainty and dependence. This would provide enough information to organize even within the sets of coupled tasks. To sequence these groups more smoothly, we would begin with the one which is missing only information that is relatively certain. Such a strategy would reduce the number of design iterations necessary in the coupled groups.

It is also likely that methods of constraint propagation $[31,36]$ can be used to help write the matrix representation automatically. This is possible only in problems that are completely described by equations, however. In principle, equation problems can be resequenced at will if numerical solution methods are acceptable [40]. That is, the equivalent of a lower triangular representation can always be found or simulated in such cases. When problems are only partially described by equations, a mixed approach is required. 
Our enhanced design structure matrix representation provides an excellent platform for this type of design research which is aimed at organizing projects with complex interactions among many engineering tasks. What makes the matrix representation successful is that it graphically exposes the difficulties (coupling) within the problem. Pracht showed that even a simple directed graph was a powerful visual aid in decision making [21]. In addition, the matrix method is well-suited to computation (although not using conventional matrix operations).

\section{Analyzing Numerical Matrix Representations}

An important advantage of the numerical design structure matrix representation is that it allows us to investigate various orderings of the tasks within a coupled block. Specifically we rank the possible sequences of the coupled tasks to choose a sequence which reduces the number of design iterations necessary to complete the coupled set of tasks.

We are developing analytical procedures which take advantage of numerical design structure matrix representations such as the one shown in Figure 4 above [29]. The numerical values convey two forms of quantitative information which can be used to evaluate the effect of coupling on the design process.

The diagonal elements indicate the time that any one design activity would consume if it were performed in isolation, with all input information available. The off-diagonal elements indicate a strength of dependence of the task on information produced by each of the other tasks. These latter values could be extracted from an engineering task/parameter sensitivity analysis.

Two potential meanings of the strength of dependence measure are being investigated. The first involves probabilities, where the numerical value indicates the probability that one additional iteration will be necessary if the interdependent tasks are performed in the specified order. Each of the dependencies is assigned one such probability, and all potential orderings of interdependent tasks are investigated in order to identify the ordering which minimizes the probability of many iterations.

The other analytical technique does not rely on a stochastic description of the design process. In this technique, the value is a measure of the portion of information produced during the first iteration which will need to be changed during the second iteration. In this way the design process can be seen as a series of iterations, each of which require a decreasing amount of time.

Through either of these methods it is possible to evaluate the degree to which the coupling in the design will affect the time necessary to complete the design. Various proposed structures of the design tasks can be compared to 
determine which would be better from a point of view of time necessary to complete the design process. We are investigating the ability of these schemes to represent (predict) design iteration in procedures with coupled tasks.

\section{Design Structure Data}

We are using the design structure matrix to represent design procedures in industry. Figure 5 is a matrix representation of the design of one component in a complex system. This matrix is based on a portion of the data we have obtained from a study at one of our sponsoring firms. The three different types of task coupling below the diagonal are labelled I, C, and M; these marks represent three different types of task dependencies: input, control, and mechanism, which are defined in the IDEFo methodology. The design tasks have been partitioned into a block-angular form which attempts to minimize the number of feedback loops. The structure suggested by this matrix is four tightly linked blocks of major iterative design tasks which are coupled through only a few of their tasks. The above-diagonal marks, labelled $F$, represent the feedback used in the design procedure to drive iteration.

The next step in our field work is to determine the numerical metrics which characterize the interactions between these design tasks. This will be accomplished by studying a particular design process [4] in enough detail to accurately document the relationships among tasks. Especially interesting will be those feedback marks which link the larger blocks of tightly coupled tasks. Redefining these inter-block constraints may provide new opportunities for innovative design management (meta-design).

As the blocks themselves represent iterations in the design process, we are using this example to study the intra-block relations using the measures described above. We believe there is tremendous advantage in performing the initial guess to start the design iteration at a specific task which may allow the design to converge quickly. This can reduce the time required by the iterative process by isolating uncertainty and increasing the confidence associated with the design decisions. 


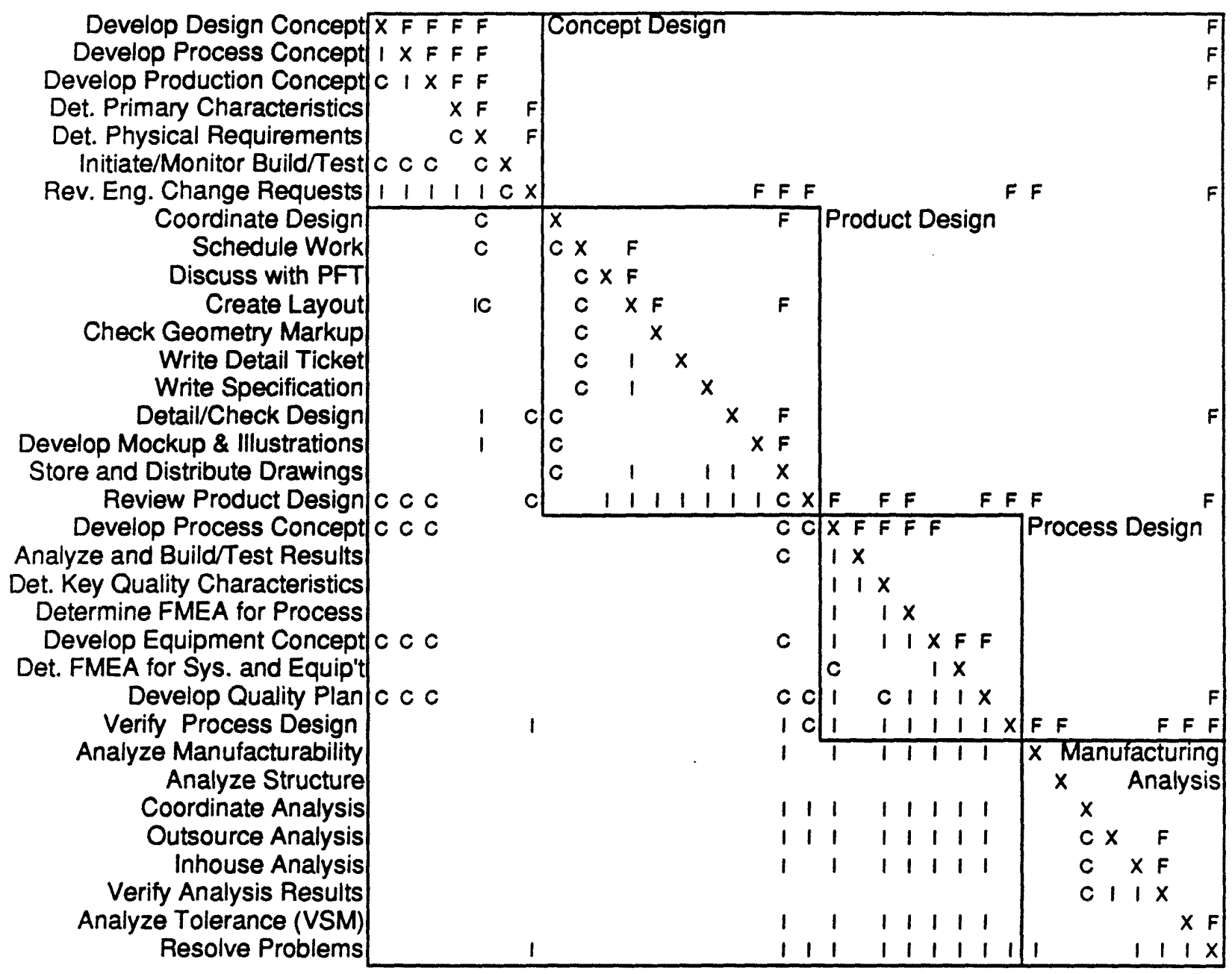

Figure 5. Data Representing an Actual Design Process

\section{Strategies for Improving the Design Process}

Analyzing the structure of a design can identify many opportunities to improve the design process. Some examples of design improvement strategies include:

Parallelization: The resequenced design process may show opportunities for newly adjacent tasks to now be performed in parallel rather than in series or as part of a large group of tasks. In Figure 3 above, the matrix decomposition shows that tasks $\mathbf{A}$ and $\mathbf{K}$ can be completed in parallel. Also task $\mathbf{G}$ can begin immediately following task $\mathbf{K}$.

Artificial Decoupling: A large, loosely coupled group of tasks can sometimes be split up into two or more smaller, more tightly coupled groups by "removing" a single task dependency (one mark in the matrix). This can be 
accomplished by scheduling an additional task to be performed earlier in the design procedure. The definition of this new task requires the parties associated with the removed dependency to agree ahead of time on the relevant task interfaces.

We also illustrate this decoupling strategy with the above matrix example. If the task dependence represented by the mark in <row $\mathbf{E}$, column $\mathbf{H}>$ can be removed by redefinition, we achieve the artificial decoupling illustrated in Figure 6. A new task has been added, task $\mathbf{X}$; its definition requires representatives from tasks $\mathbf{H}, \mathbf{D}$, and $\mathbf{E}$ to specify their new task interfaces so that they can complete their work independently. Note that defining the new task may require even more task-structure information than we have available; however, application of this strategy can have a tremendous impact on overall project performance.

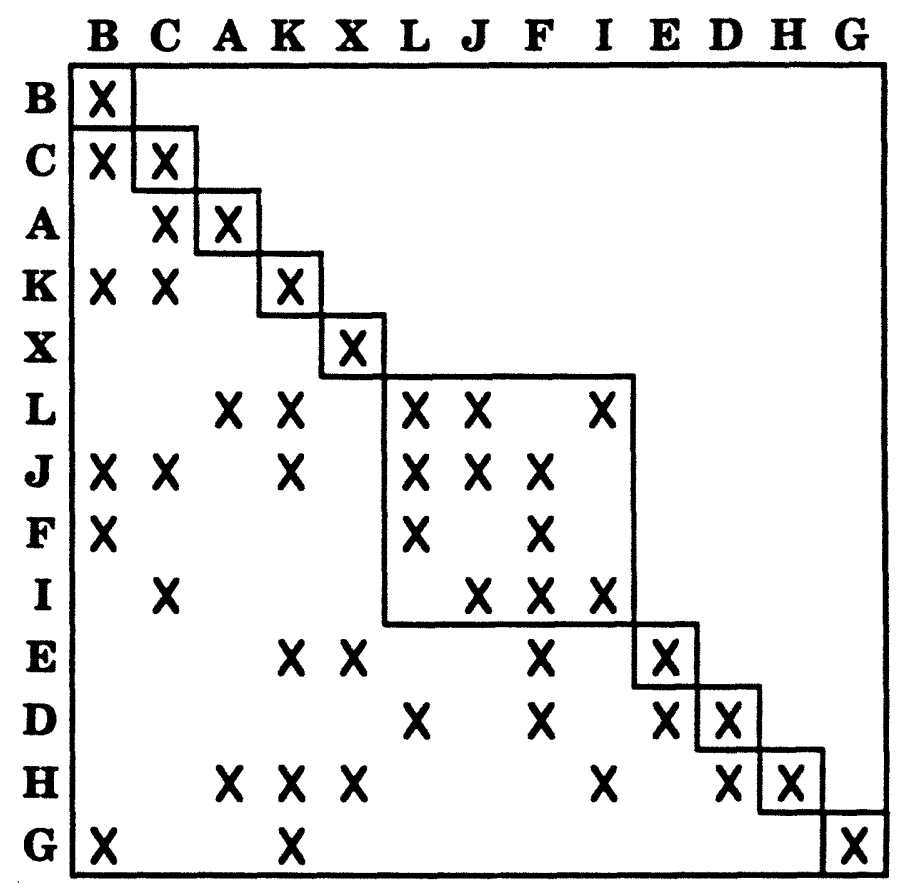

Figure 6. Artificial Decoupling

Increased Coupling: This strategy is the essential basis of simultaneous engineering and design for manufacture. The performance of design engineering and manufacturing engineering concurrently requires a tremendous volume of information transfer. According to our models, this increases task coupling and impedes the design process. What is missing in our representation is a measure of output design quality, which design for manufacturing intends to improve. 


\section{Conclusion}

The results of this research promise to be new tools and attitudes applicable to improving the design process. In contrast to traditional project management tools, this one explicitly takes account of the technical structure and facts of the problem, allowing more detailed and specific improvements to be made. (PERT/CPM in fact is not aimed at improving the process but rather only at running the project as is.)

While it is often assumed that tools like the design structure method are most applicable to new designs (Rogers), we have found two reasons why this work can have an even larger impact on designs with existing procedures that badly need improvement. First, our documentation of an existing process can reveal surprising features that our tools will clean up. We can help managers to recognize ways in which the process can be streamlined. Use of the tools gives managers a way to document existing processes that did not exist before and help them structure the very process of documentation itself by providing a framework and terminology for doing so.

Second, industry is entering the phase of "concurrent design", in which new design processes must be created as old ones are discarded. New players are entering the design game earlier and raising issues once left for later. When is the best time to consider them? Not everything can be "simultaneous" in simultaneous engineering!

Elaborating the types of interactions and interdependencies that actually exist in real design processes via our case studies will help guide this research, making it more realistic and applicable to conditions in industry. In this way we may discover that the matrix representation, while an improvement over others, is nonetheless a simplification that needs improvement itself. Thus the research will impact further research.

This paper describes ongoing research. We are currently engaged in activities aimed at the following major objectives:

1. Develop improved design representation models by conducting design process case studies documenting design procedures and their underlying engineering structure.

2. Develop a computer-based analytical interactive tool that operates upon the design structure models and helps design managers find ways to improve the organization of complex design projects.

3. Develop technical and organizational strategies that exploit the power of the analytical tool to identify ways to reduce the complexity of concurrent product/process development efforts. 


\section{Bibliography}

[1] C. Alexander. Notes on the Synthesis of Form. Harvard University Press, Cambridge, Massachusetts, 1964.

[2] T. Allen. Managing the Flow of Technology: Technology Transfer and the Dissemination of Technological Information Within the R\&D Organization. MIT Press, Cambridge, MA, 1977.

[3] D. G. Ancona and D. E. Caldwell. "Demography and Design: Predictors of New Product Team Performance", Working Paper, MIT Sloan School of Management. September 1989, no. 3078-89.

[4] T. A. Black. A Systems Design Methodology Applied to Automotive Brake Design. MIT S.M. Thesis, 1990.

[5] A. H. Bond and R. J. Ricci. Cooperation in Aircraft Design. MIT-JSME Workshop on Cooperative Product Development, Cambridge, MA, November 1989,

[6] G. Boothroyd and P. Dewhurst. Product Design for Assembly. Boothroyd Dewhurst, Inc., Wakefield, RI, 1987.

[7] L. L. Bucciarelli. "An Ethnographic Perspective on Engineering Design", Design Studies. vol. 9, no. 3, July 1988, pp. 159-168.

[8] D. M. Byrne and S. Taguchi. "The Taguchi Approach to Parameter Design", Quality Progress. December 1987, pp. 19-26.

[9] K. B. Clark. "Project Scope and Project Performance: The Effect of Parts Strategy and Supplier Involvement on Product Development", Management Science. vol. 35, no. 10, October 1989, pp. 1247-1263.

[10] K. B. Clark, B. Chew and T. Fujimoto. "Product Development in the World Auto Industry", Brookings Papers on Economic Activity. vol. 3, 1989, pp. 729-771.

[11] J. W. Dean Jr. and G. I. Susman. "Organizing for Manufacturable Design", Harvard Business Review. January-February 1989, pp. 28-36.

[12] P. F. Drucker. "The Discipline of Innovation", Harvard Business Review. May-June 1985, pp. 67-72.

[13] T. C. Hartrum, T. D. Connally and S. E. Johnson. An Interactive Graphics Editor with Integrated Data Dictionary for IDEF ${ }_{0}$ Structured Analysis Diagrams. National Aerospace and Electronics Conference, Dayton, Ohio, May 1988, pp. 765-770.

[14] J. R. Hauser and D. Clausing. "The House of Quality", Harvard Business Review. May-June 1988, pp. 63-73. 
[15] T. Kitzmiller. Avionic System Design. MIT-JSME Workshop on Cooperative Product Development, Cambridge, MA, November 1989,

[16] W. P. Ledet and D. M. Himmelblau. "Decomposition Procedures for the Solving of Large Scale Systems", Advances in Chemical Engineering. vol. 8, pp. 185-254.

[17] H. L. Malchow and S. R. Croopnick. "A Methodology for Organizing Performance Requirements for Complex Dynamical Systems", IEEE Transactions on Engineering Management. February 1985,

[18] D. A. Marca and C. L. McGowan. SADT: Structured Analysis and Design Technique. McGraw Hill, New York, 1988.

[19] J. L. Nevins and D. E. Whitney. Concurrent Design of Products and Processes. McGraw-Hill, New York, 1989.

[20] S. L. Padula, C. Sandridge, R. T. Haftka and J. L. Walsh. "Demonstration of Decomposition and Optimization in the Design of Experimental Space Systems". In J.-F. M. Barthelemy, Ed. Recent Advances in Multidisciplinary Analysis, NASA Langley Research Center, Hampton, Virginia, 1988.

[21] W. E. Pracht. "Gismo: A Visual Problem-Structuring and KnowledgeOrganization Tool", IEEE Transactions on Systems, Man, and Cybernetics. vol. SMC-16, no. 2, March/April 1986, pp. 265-270.

[22] J. B. Quinn. "Managing Innovation: Controlled Chaos", Harvard Business Review. May-June 1985, pp. 73-84.

[23] J. R. Rinderle and N. P. Suh. "Measures of Functional Coupling in Design", ASME Journal of Engineering for Industry. November 1982, pp. 383-388.

[24] J. L. Rogers. DeMAID: A Design Manager's Aide for Intelligent Decomposition User's Guide. NASA Technical Memorandum 101575, March 1989.

[25] J. L. Rogers and S. L. Padula. An Intelligent Advisor for the Design Manager. NASA Technical Memorandum 101558, February 1989.

[26] D. T. Ross. "Structured Analysis (SA): A Language for Communicating Ideas", IEEE Transactions on Software Engineering. vol. SE-3, no. 1, January 1977, pp. 16-34.

[27] D. T. Ross. "Applications and Extensions of SADT", IEEE Computer Magazine. April 1985, pp. 25-34.

[28] H. A. Simon. The Sciences of the Artificial. MIT Press, Cambridge, Massachusetts, 1970. 
[29] R. P. Smith and S. D. Eppinger. "Modeling Design Iteration", Working Paper, MIT Sloan School of Management. June 1990, no. 3160-90-MS.

[30] J. Sobieszczanski-Sobieski. Multidisciplinary Optimization for Engineering Systems: Achievements and Potential. NASA Technical Memorandum 101566, March 1989.

[31] D. Sriram and M. L. Maher. "Representation and Use of Constraints in Structural Design", AI in Engineering. Springer-Verlag, Southampton, UK, April 1986.

[32] D. V. Steward. "The Design Structure System: A Method for Managing the Design of Complex Systems", IEEE Transactions on Engineering Management. August 1981, pp. 71-74.

[33] D. V. Steward. Systems Analysis and Management: Structure, Strategy, and Design. Petrocelli Books, New York, 1981.

[34] N. P. Suh. "Basic Concepts in Design for Producibility", Annals of the CIRP. vol. 32, no. 2, 1988, pp. 559-566.

[35] N. P. Suh, A. C. Bell and D. C. Gossard. "On an Axiomatic Approach to Manufacturing and Manufacturing Systems", ASME Journal of Engineering for Industry. May 1978, pp. 127-130.

[36] G. J. Sussman and G. L. Steele. "Constraints -- A Language for Expressing Almost-Hierarchical Descriptions", Artificial Intelligence. vol. 14, 1980, pp. 1-39.

[37] E. von Hippel. Task Partitioning: An Innovation Process Variable. MIT Sloan School of Management Working Paper no. 2030-88, June 1988 (rev. April 1989).

[38] J. N. Warfield. "Binary Matrices in System Modeling", IEEE Transactions on Systems, Man, and Cybernetics. vol. SMC-3, no. 5, September 1973, pp. 441-449.

[39] D. E. Whitney. "Manufacturing By Design", Harvard Business Review. July-August 1988, pp. 83-91.

[40] D. E. Whitney and M. Milley. "CADSYS: A New Approach to ComputerAided Design", IEEE Transactions on Systems, Man, and Cybernetics. vol. SMC-4, no. 1, January 1974, pp. 50-58.

[41] J. D. Wiest. "Precedence Diagramming Method: Some Unusual Characteristics and Their Implications for Project Managers", Journal of Operations Management. vol. 1, no. 3, February 1981, pp. 121-130.

[42] J. D. Wiest and F. K. Levy. A Management Guide to PERT/CPM. PrenticeHall, Englewood Cliffs, New Jersey, 2nd Edition, 1977. 